\title{
SOLAR OBSERVATIONS WITH LOFAR
}

\author{
G. Mann*, C. Vocks*, and F. Breitling*
}

\begin{abstract}
The LOw Frequency ARray (LOFAR) is a novel radio telescope that consists of 20 core stations near Exloo in the Netherlands, 18 Dutch remote stations, and 8 international stations all distributed over central Europe. One of them was built in Potsdam. LOFAR is a radio interferometer for the frequency range of $30-240 \mathrm{MHz}$ and will exceed the sensitivity and resolution of existing instruments by more than one order of magnitude. "Solar Physics and Space Weather with LOFAR" is one of LOFAR's Key Science Projects. Since LOFAR is working in the frequency range of $30-240 \mathrm{MHz}$, it is able to deliver radio images of the Sun. The solar radio radiation in this frequency range is emitted from the outer corona. Since radio waves are strongly scattered in the corona, the angular resolution of LOFAR's radio maps will be limited to a few 10 arcseconds. The necessary baselines include the core and the nearest remote stations.
\end{abstract}

\section{Introduction}

The Sun is an intense radio source in sky. Especially, during solar eruptions as flares and coronal mass ejections (CME), the intensity of solar radio emission is strongly enhanced. The radio emission of the Sun covers a broad spectrum from the $\mathrm{GHz}$ range down to the $\mathrm{MHz}$ range (see McLean and Labrum [1985] as a review book).

LOw Frequency ARray (LOFAR) is originally designed by ASTRON in the Netherlands to observe extraterrestrial objects at low frequencies, i.e. $30-240 \mathrm{MHz}$. It consists of 20 core and 18 remote stations in the Dutch and, additionally, 8 remote stations in Europe as Sweden, UK, France, and Germany as well as possibly more in Spain and Poland in future. In Germany, 5 stations have been installed. One of them is located in Potsdam (Fig. 1) and belongs to the Leibniz-Institut für Astrophysik Potsdam (AIP). LOFAR is an interferometer and is designed to deliver radio images of astronomical objects in sky, as the Sun for instance.

The core science program of LOFAR is organized in 6 so-called Key Science Projects (KSP). One of them, "Solar Physics and Space Weather with LOFAR" is coordinated by

* Astrophysikalisches Institut Potsdam, An der Sternwarte 16, 14482 Potsdam, Germany 


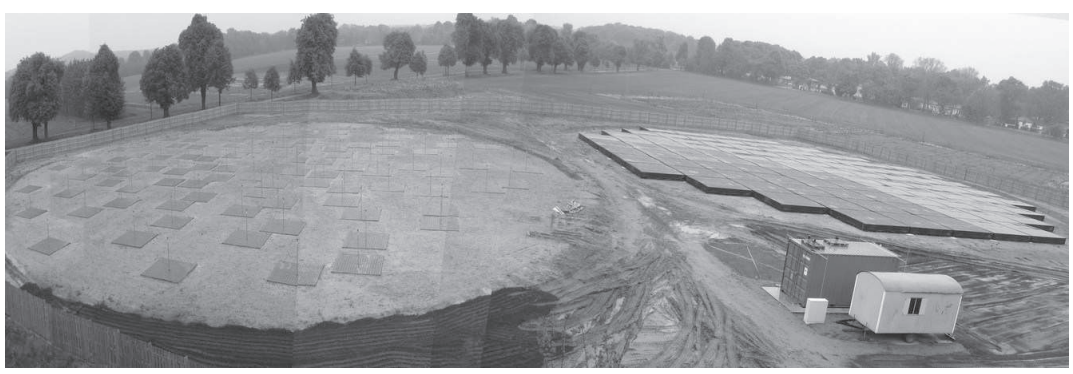

Figure 1: The LOFAR remote station of the AIP in Potsdam-Bornim. The LBA and HBA field are on the left and right, resp..

the AIP. 31 scientists from 11 European countries participate in the solar KSP. Detailed information on this KSP can be found in web page under http://www.aip.de/groups/osra/sksp/.

With LOFAR, a novel technology is realized. An ensemble (presently 46) of LOFAR stations are distributed in the Netherlands and Europe. The radio signals from each station are transferred via a $10 \mathrm{Gbit} / \mathrm{s}$ data link to the BlueGene super-computer in Groningen, where they are correlated to a radio map of the sky. Each LOFAR station has 96 low (LBA) (Fig. 2) and 96 high band antennas (HBA) (Fig. 3) for the frequency ranges 30-80 and 120-240 MHz. respectively. Each HBA antenna comprises 16 dipoles, which are used jointly to form a beam.

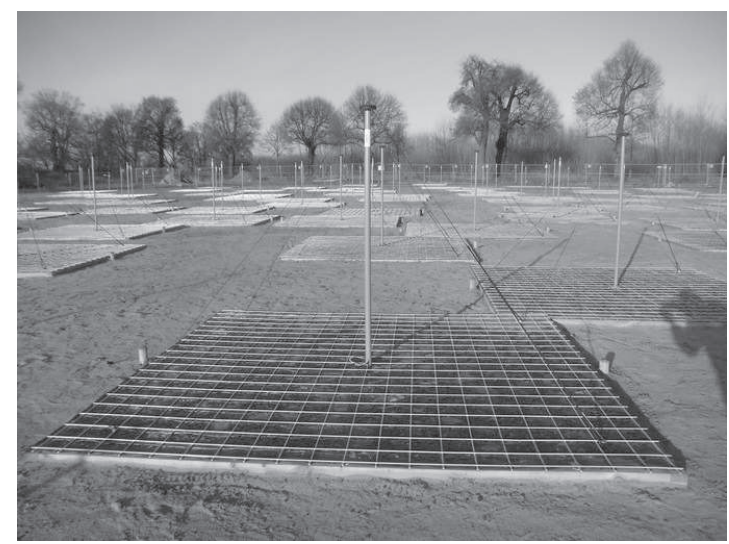

Figure 2: A single low band antenna (30-80 MHz). 


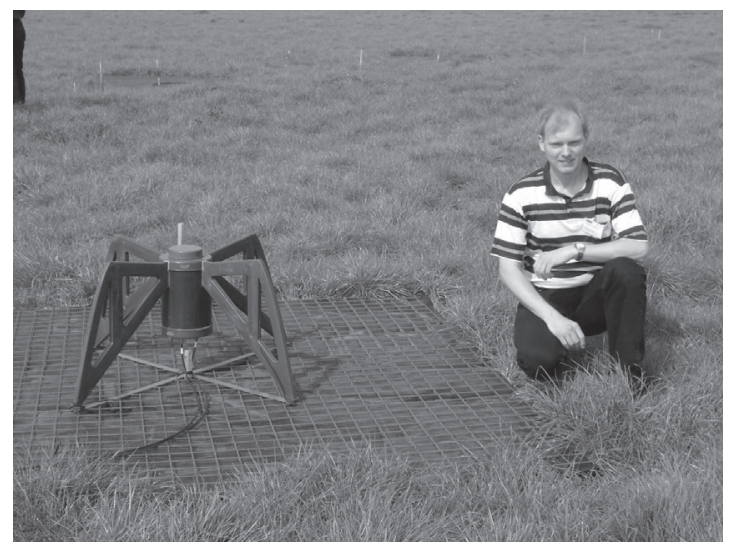

Figure 3: One of the 16 dipoles which form a high band antenna (120-240 MHz).

\section{Solar Radio Emission}

During solar flares the solar radio emission is strongly enhanced. That is seen in Figure 4 for instance. It shows the dynamic radio spectrum in the range $200-400 \mathrm{MHz}$ as recorded by the radio spectral-polarimeter [Mann et al., 1992] of the AIP during the solar flare on October 28, 2003. It was one of the hugest events, which were ever observed. The flare starts with a strong enhancement of radio emission at $300 \mathrm{MHz}$ on $11 \mathrm{UT}$. One minute later, the spectrum of the emitted radio waves becomes much broader. Simultaneously, an enhancement of the hard X-ray radiation, i.e. for photons with energies $>150 \mathrm{keV}$ is seen by the RHESSI satellite. Only during the first minute of this event, the emission of $\gamma$-rays is also enhanced in the energy range $7.5-10 \mathrm{MeV}$ as seen by the INTEGRAL spacecraft. That demonstrates, that there is a strong correlation between the non-thermal radio, hard X-ray, and $\gamma$-ray emission. For all of theses cases, energetic electrons are necessary. That indicates the generation of energetic electrons during solar flares. A detailed data analysis revealed that $\approx 10^{36}$ electrons with energies $>20 \mathrm{keV}$ are produced within a second during large flares [Warmuth et al., 2009].

During solar flares, the Sun emits nonthermal radio radiation. In the frequency range below $1 \mathrm{GHz}$, it is thought to be generated by plasma and/or gyrosynchrotron emission. In the case of plasma emission, the radio waves are emitted near the local plasma frequency and/or its harmonics [Melrose, 1985]. The electron plasma frequency is depending on the square root of the electron number density. Due to the gravitational stratification of the solar atmosphere, the high and low frequency radio waves are emanated from the low and high corona, respectively. Or in other words, each frequency corresponds to a certain height level in the corona. That is not totally true in the case of the quiet Sun, since the radio emission is bremsstrahlung for this case. Here, the low-frequency emission can come from greater heights than from the simple height-frequency relationship.

The corona is highly structured in space and variable in time. Koutchmy [1994] reports 


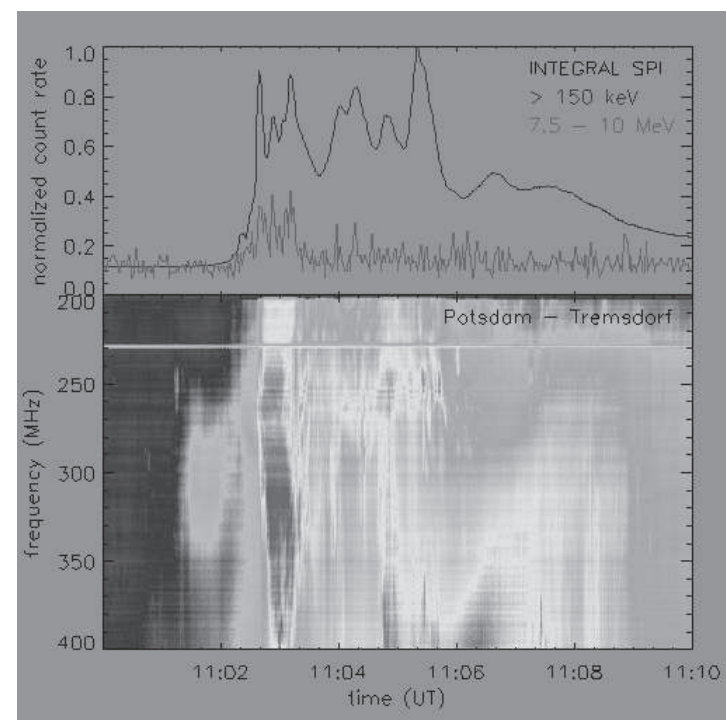

Figure 4: The dynamic radio spectrum (bottom) in the range 200-400 $\mathrm{MHz}$ during the onset of the solar event on October 28, 2003. The radio intensity is color coded. At top, the temporal behavior of the photon count rates is shown for energies $>150 \mathrm{keV}$ (hard X-rays, upper curve) and 7.5-10 MeV ( $\gamma$-rays, lower curve).

on remote-sensing measurements of the electron number density in the corona. But the height dependence of the density is approximately described by an barometric height formula (Mann et al. 1999)

$$
N(r)=N_{\odot} \cdot \exp \left(\lambda\left[\frac{R_{\odot}}{r}\right]-1\right)
$$

with $\lambda=\lambda_{0} \cdot\left(T_{0} / T\right)$ and $\lambda_{0}=\tilde{\mu} \gamma_{G} m_{p} M_{\odot} / k_{B} T_{0} R_{\odot}=13.83$ (N, electron number density; $\mathrm{k}_{B}$, Boltzmann's constant; $\mathrm{T}$, temperature; $\tilde{\mu}$, mean molecular weight; $\mathrm{m}_{p}$, proton mass; $\gamma_{G}$, gravitational constant; $M_{\odot}$, mass of the Sun; $R_{\odot}$, radius of the Sun) for $\tilde{\mu}=0.6$ (see Priest [1982]) and $T_{0}=10^{6} \mathrm{~K}$. Here, $N_{\odot}$ denotes the electron number density at the bottom of the corona. It can vary over three orders of magnitude. Eq. (1) agrees with the one-fold Newkirk [1961] model for $N_{\odot}=8.78 \times 10^{8} \mathrm{~cm}^{-3}$ and a temperature $\mathrm{T}=1.4$ $\times 10^{6} \mathrm{~K}$. Such a model can be considered as an appropriate one for quiet coronal regions [Koutchmy, 1994]. According to Eq. (1), each density and, consequently, each frequency corresponds to a certain height level in the corona.. Thus, LOFAR is able to observe the corona up to a distance of $\approx 1.8 \mathrm{R}_{\odot}$ from the center of the Sun (or to a height of $560 \mathrm{Mm}$ above the photosphere). Hence, the solar activity can be monitored up to a distance of $\approx 1.8 \mathrm{R}_{\odot}$ by LOFAR. Because of these reasons, LOFAR is a very interesting facility for solar observations and, consequently, for solar physics. Since CMEs are launched in the outer corona and they are influencing our Earth's environment, if they hit the Earth, their observation by LOFAR is also interesting as a service for Space Weather. 


\section{Solar Observation Modes with LOFAR}

LOFAR will be able to measure the solar radio radiation in the range $30-240 \mathrm{MHz}$ with a high spatial, temporal, and spectral resolution. The radio images at different frequencies, i.e. at different height levels, allows a 3D tomography of the flare related processes in the corona of the Sun.

The participants of the solar KSP proposes the following observing modes with LOFAR:

- dynamic radio spectrometry (spectrometer mode)

- monitoring the solar activity (monitoring mode)

- solar burst imaging (burst mode)

In the case of the spectrometer mode, a single LOFAR station is used as a pure radio spectrometer. Here, the 165 sub-bands with $195 \mathrm{kHz}$ bandwidth are evenly distributed over the whole low and high band range. The temporal resolution should be at least $10 \mathrm{~ms}$ in order to reveal fine structures in solar radio bursts. That would lead to a data rate of $0.34 \mathrm{MB} / \mathrm{s}$. Of course, LOFAR is a radio interferometer basically designed as an imaging instrument. But it can only work in such way if all stations are connected with each other and are operated collectively. In the stand-alone case, a single station could potentially be used as a spectrometer.

The monitoring mode is thought to be a input service for the Space Weather community. Routine monitoring of the Sun comprises aperture synthesis images of the Sun at several frequencies both in the low and high bands, e.g. at 40, 80, 160, and $240 \mathrm{MHz}$. The imaging cadence is proposed to be 1 image per minute at each of these four frequencies in order to link to the US $\mathrm{H}_{\alpha}$-patrol mission. This mode produces a data rate of $0.03 \mathrm{MB} / \mathrm{s}$.

Solar radio burst studies comprise a rapid sequence of aperture synthesis images of the Sun. Such observations would be complementary to space missions like RHESSI, Hinode, and SDO (Solar Dynamic Observatory). The burst mode requires an imaging sequence of at least $0.1 \mathrm{~s}$ in order to cover the whole dynamic evolution of the burst. It is proposed to take the images simultaneously in steps of 5 and $10 \mathrm{MHz}$ in the low $(30-80 \mathrm{MHz})$ and high (120-240 MHz) band, respectively. That would get a data rate of $100 \mathrm{MB} / \mathrm{s}$. The burst mode is intended to be performed in terms of so-called campaigns with a duration of several weeks. They should be coordinated with space-born and ground-based solar observations.

\section{Concluding Remarks}

With LOFAR, the processes, which are related to energetic electrons in the solar corona, can be observed with a high spatial and temporal resolution. These processes are of special interest, since they are influencing our Earth's environment and our technical civilization. That is usually called Space Weather. That demonstrates that LOFAR is a very interesting facility for the solar physics and Space Weather community. 


\section{References}

Koutchmy, S., Coronal physics from eclipse observations, Adv. Space Res., 14, (4)29(4)39, 1994.

Mann, G., H. Aurass, H. Voigt, and J. Paschke, Preliminary observations of solar type II bursts with the new radio spectrograph in Tremsdorf (Germany), ESA SP-348, 129-135, 1992.

Mann, G., F. Jansen, R. J. MacDowall, M. L. Kaiser, and R. G. Stone, A heliospheric density model and type III bursts, Astron. Astrophys., 348, 614-621, 1999.

McLean, D. J., and N.R. Labrum, Solar Radiophysics, Cambridge Univ. Press, Cambridge, 1985.

Melrose, D. B., Plasma emission mechanisms, in Solar Radiophysics, edited by D. J. McLean and N. R. Labrum, Cambridge Univ. Press, Cambridge, 177-210, 1985.

Newkirk, Jr. G., The solar corona in active regions and the thermal origin of the slowly varying component of solar radio radiation, Astrophys. J., 133, 983-996, 1961.

Priest, E. R., Solar Magnetohydrodynamics, Reidel, Dordrecht, 1982.

Warmuth, A., G. Mann, and H. Aurass, Modelling shock drift acceleration of electrons at the reconnection outflow termination shock in solar flares, Astron. Astrophys., 494, 677-691, 2009. 